\title{
Fe II EMISSION IN ACTIVE GALACTIC NUCLEI: THE ROLE OF TOTAL AND GAS-PHASE IRON ABUNDANCE
}

\author{
Gregory A. Shields ${ }^{1}$, RAndi R. Ludwig ${ }^{1}$, And SARAh SAlviander ${ }^{1,2}$ \\ ${ }^{1}$ Department of Astronomy, University of Texas, Austin, TX 78712, USA; shields@astro.as.utexas.edu, randi@ astro.as.utexas.edu, triples@astro.as.utexas.edu \\ 2 Department of Physics, Southwestern University, Georgetown, TX 78626, USA \\ Received 2010 June 9; accepted 2010 August 5; published 2010 September 14
}

\begin{abstract}
Active galactic nuclei (AGNs) have Fe II emission from the broad-line region (BLR) that differs greatly in strength from object to object. We examine the role of the total and gas-phase iron abundance in determining Fe II strength. Using AGN spectra from the Sloan Digital Sky Survey (SDSS) in the redshift range of $0.2<z<0.35$, we measure the $\mathrm{Fe} / \mathrm{Ne}$ abundance of the narrow-line region (NLR) using the $[\mathrm{Fe} \mathrm{VII}] /[\mathrm{Ne} \mathrm{v}]$ line intensity ratio. We find no significant difference in the abundance of Fe relative to Ne in the NLR as a function of $\mathrm{Fe}$ II $/ \mathrm{H} \beta$. However, the $[\mathrm{NII}] /[\mathrm{S} \mathrm{II}]$ ratio increases by a factor of 2 with increasing Fe II strength. This indicates a trend in N/S abundance ratio, and by implication in the overall metallicity of the NLR gas, with increasing Fe II strength. We propose that the wide range of Fe II strength in AGN largely results from the selective depletion of Fe into grains in the low ionization portion of the BLR. Photoionization models show that the strength of the optical Fe II lines varies almost linearly with gas-phase Fe abundance, while the ultraviolet Fe II strength varies more weakly. Interstellar depletions of $\mathrm{Fe}$ can be as large as 2 orders of magnitude, sufficient to explain the wide range of optical Fe II strength in AGNs. This picture is consistent with the similarity of the BLR radius to the dust sublimation radius and with indications of Fe II emitting gas flowing inward from the dusty torus.
\end{abstract}

Key words: galaxies: active - quasars: general

\section{INTRODUCTION}

The broad emission-line spectrum of quasars often includes strong Fe II in the optical and ultraviolet. The difference between the weakest and strongest optical Fe II emission exceeds a factor of 10, measured as equivalent width (EW) or as Fe II/ $\mathrm{H} \beta$ line ratio. For recent discussions of $\mathrm{Fe}_{\mathrm{II}}$ intensities in active galactic nuclei (AGNs), and references to earlier work, see Kovačević et al. (2010) and Ferland et al. (2009). The Fe II strength anti-correlates with the strength of the narrow [O III] emission line. This trend along with several associated correlations defines the so-called Eigenvector 1 (EV1), which characterizes some of the most conspicuous differences among the properties of AGNs (Boroson \& Green 1992, hereinafter BG92). The quest for physical drivers of EV1 has inspired a number of studies (e.g., Wills et al. 1999; Marziani et al. 2003). Boroson (2002) and Netzer \& Trakhtenbrot (2007) find that Fe II increases with Eddington ratio $L / L_{\mathrm{Ed}}$, as originally suggested by BG92. However, the physics underlying this correlation remains unclear. There is even debate as to whether the Fe II emission is entirely powered by the ionizing continuum of the central source, or comes in some measure from a mechanically heated region (Wills et al. 1985; Collin \& Joly 2000; Sigut \& Pradhan 2003; Bruhweiler \& Verner 2008). In this situation, any observational clues to the nature of EV1 and the great range of Fe II strength are of value.

It is generally assumed that the strength of Fe II emission is driven by physical conditions within the broad-line region (BLR), such as ionizing continuum, BLR density and geometry, column density, and turbulent velocity. However, high abundance of $\mathrm{Fe}$ has been discussed to help produce the strongest Fe II observed (Wills et al. 1985; Collin-Souffrin et al. 1988; Hamann \& Ferland 1993). The utility of Fe II to assess the Fe abundance in high redshift QSOs has received considerable interest, in the context of galactic chemical evolution (Hamann \& Ferland 1993; Verner et al. 2003; Baldwin et al. 2004; Netzer \&
Trakhtenbrot 2007, and references therein). Here we assess the importance of the abundance of Fe relative to the $\alpha$-elements, the overall metallicity of the nuclear gas, and the depletion of $\mathrm{Fe}$ into grains for the strength of $\mathrm{Fe}$ II emission in QSOs.

\section{QSO SAMPLE AND MEASUREMENTS}

We investigated the influence of differing abundances in quasars on their optical Fe II emission strength by studying the optical emission-line properties of a sample of QSOs from the Sloan Digital Sky Survey (SDSS). ${ }^{3}$ It is difficult to measure abundances within the BLR directly, because it is a region of high density, line width, and line optical depth. On the assumption that the abundance in the narrow-line region (NLR) and BLR is the same for a given object (see below), we used the narrow emission-line spectrum to assess abundances in the NLR. We considered two key line ratios: (1) the [Fe VII]/ $[\mathrm{Nev}]$ intensity ratio gives a measure the $\mathrm{Fe} / \mathrm{Ne}$ abundance ratio, and (2) the $[\mathrm{N} \mathrm{II}] /[\mathrm{S} \mathrm{II}]$ ratio gives a measure of the $\mathrm{N} /$ $\mathrm{S}$ abundance ratio, which is in turn a secondary indicator of the overall metallicity of the gas. Our sample consists of 1571 quasars from SDSS Data Release 7 (DR7). These objects were selected in the manner of the "HO3" sample of Salviander et al. (2007), with the additional requirement of a signal-to-noise ratio $(\mathrm{S} / \mathrm{N})$ greater than 10 in the continuum at $\lambda 5100$ rest wavelength. The flux and EW of the broad $\mathrm{H} \beta$ line and the optical $\mathrm{Fe}$ II blends were measured with the aid of a spectrum fitting program described by Salviander et al. (2007), using a template fitting procedure to establish the flux in Fe II relative to the local continuum. We characterized the Fe II emission strength using the flux ratio of the Fe II $4570 \AA$ blend to broad $\mathrm{H} \beta$, following BG92. We used quasars at redshifts $0.2<z<0.35$ to ensure coverage of both [Ne v] $\lambda 3425$ and [Fe VII] $\lambda 6087$. In order to bring out the weak [Fe VII] line, we binned the 1571

\footnotetext{
3 The SDSS Web site is http://www.sdss.org.
} 


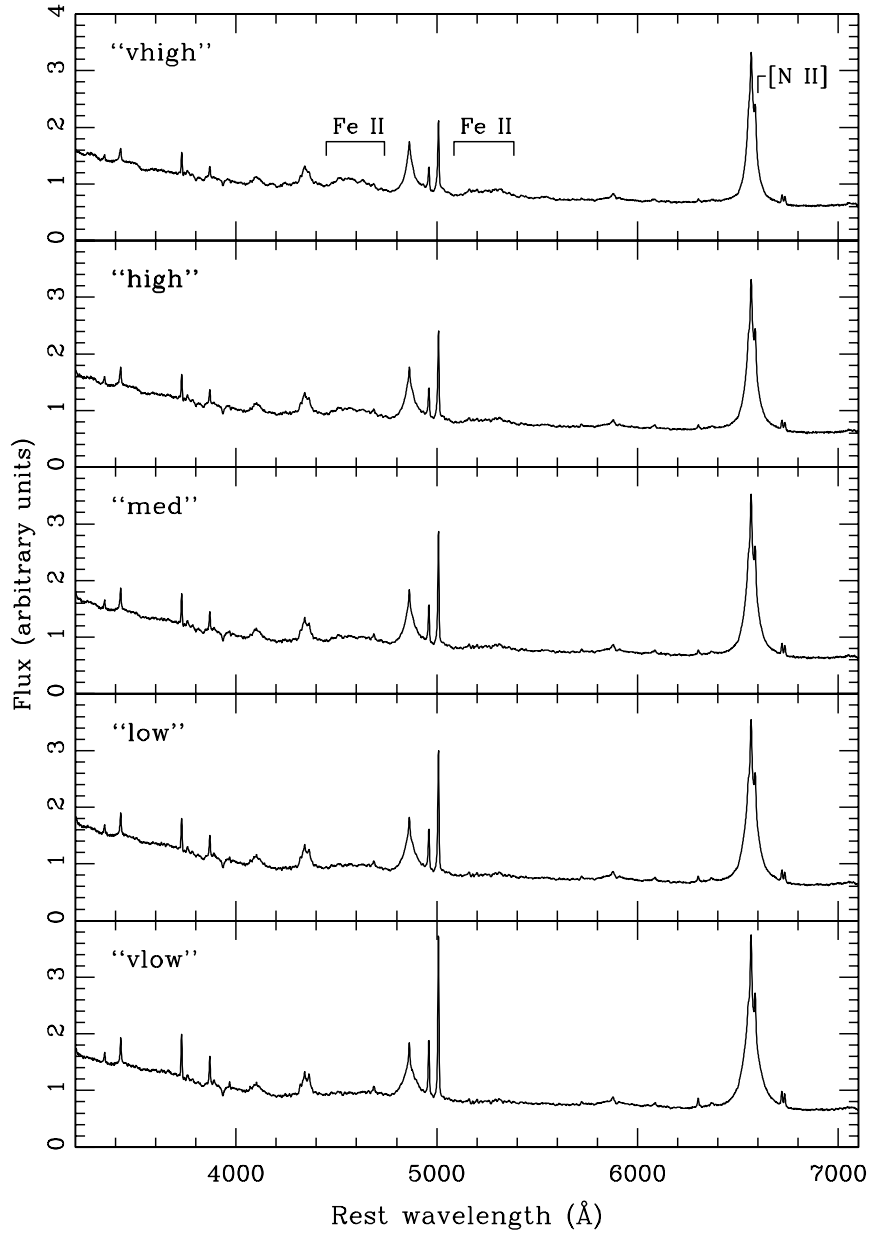

Figure 1. Composite SDSS spectra for the five bins in optical Fe II strength. Vertical axis gives specific flux $F_{\lambda}$. Note the broad Fe II blends at $\lambda 4570$ and $\lambda 5250$. See the text for discussion.

objects by Fe II strength, and made five composite spectra of "very weak," "weak," "medium," "strong," and "very strong"
Fe II emission. These composites had 312 to 315 objects, within bins bounded by $\mathrm{Fe}$ II $/ \mathrm{H} \beta$ values of $0,0.215,0.372,0.524$, 0.708 , and 1.82 , respectively. Individual spectra were corrected for Galactic reddening using the extinction values $A_{g}$ given by the SDSS pipeline, and normalized to a mean flux density $F_{\lambda}$ of unity using all wavelength points in a particular spectrum. The individual spectra in each group were shifted in wavelength to the rest frame and re-binned to a common wavelength grid at a spacing of $1.41 \AA$. The adopted composite spectrum was a mean of the rebinned $F_{\lambda}$ for all contributing spectra at a given wavelength. The composite spectra are shown in Figures 1 and 2. The region of the $[\mathrm{Fe} \mathrm{VII}]$ and $[\mathrm{Ne} \mathrm{V}]$ line is shown in Figure 2. For a discussion of issues involving composite spectra of QSOs, see Vanden Berk et al. (2001), and references therein.

From these composites, we measured the emission-line fluxes of a number of lines, including [O III] $\lambda 5007$, [O II] $\lambda 3727,[\mathrm{Ne}$ III] $\lambda 3869$, [Ne v] $\lambda 3425$, [S II] $\lambda \lambda 6716,6730$, and $[\mathrm{Fe}$ VII $] \lambda 6087$. The results are given in Table 1 and Figure 3, where the $\mathrm{Fe} I \mathrm{I} / \mathrm{H} \beta$ values are averages of the values for the individual spectra that compose each composite. Most lines were measured using a Gaussian fit with the IRAF task SPLOT. ${ }^{4}$ The broad Balmer emission lines $(\mathrm{H} \alpha, \mathrm{H} \beta)$ were measured using a Lorentzian profile. Estimated uncertainties are $10 \%$ for the stronger lines, including continuum placement and faithfulness of the fit. For [Fe VII] and [Ne v], the uncertainty is as much as $20 \%$, based on noise, continuum uncertainty, and the presence of a strong blue wing on both lines that we excluded from the fit. The $\left[\mathrm{N}_{\mathrm{II}}\right] \lambda 6583$ and $\lambda 6548$ lines were fairly weak bumps on the wings of the broad $\mathrm{H} \alpha$ line and relatively difficult to measure. Therefore, we measured the $[\mathrm{N}$ II] intensity by subtracting from the $\mathrm{H} \alpha-[\mathrm{N}$ II $]$ blend a doublet with the theoretical 3.0-to-1 intensity ratio, each line having a Gaussian profile with a central wavelength and width based on the redshift and line width of [S II] $\lambda 6716$. The intensity of the doublet was adjusted so that the $\mathrm{H} \alpha$ line had a smooth profile with no visible

\footnotetext{
4 IRAF is distributed by the National Optical Astronomy Observatories, which are operated by the Association of Universities for Research in Astronomy, Inc., under cooperative agreement with the National Science Foundation.
}

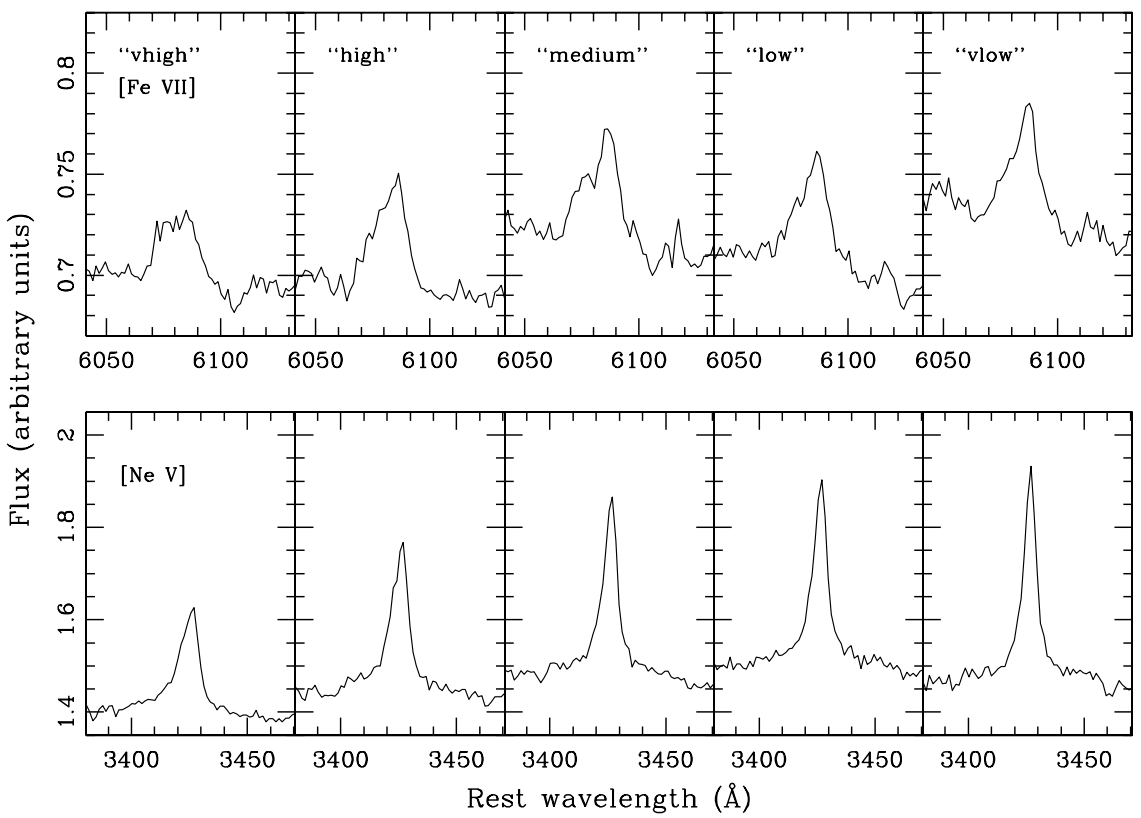

Figure 2. [Fe VII] and [Ne v] narrow emission lines in the composite spectra. See the text for discussion. 


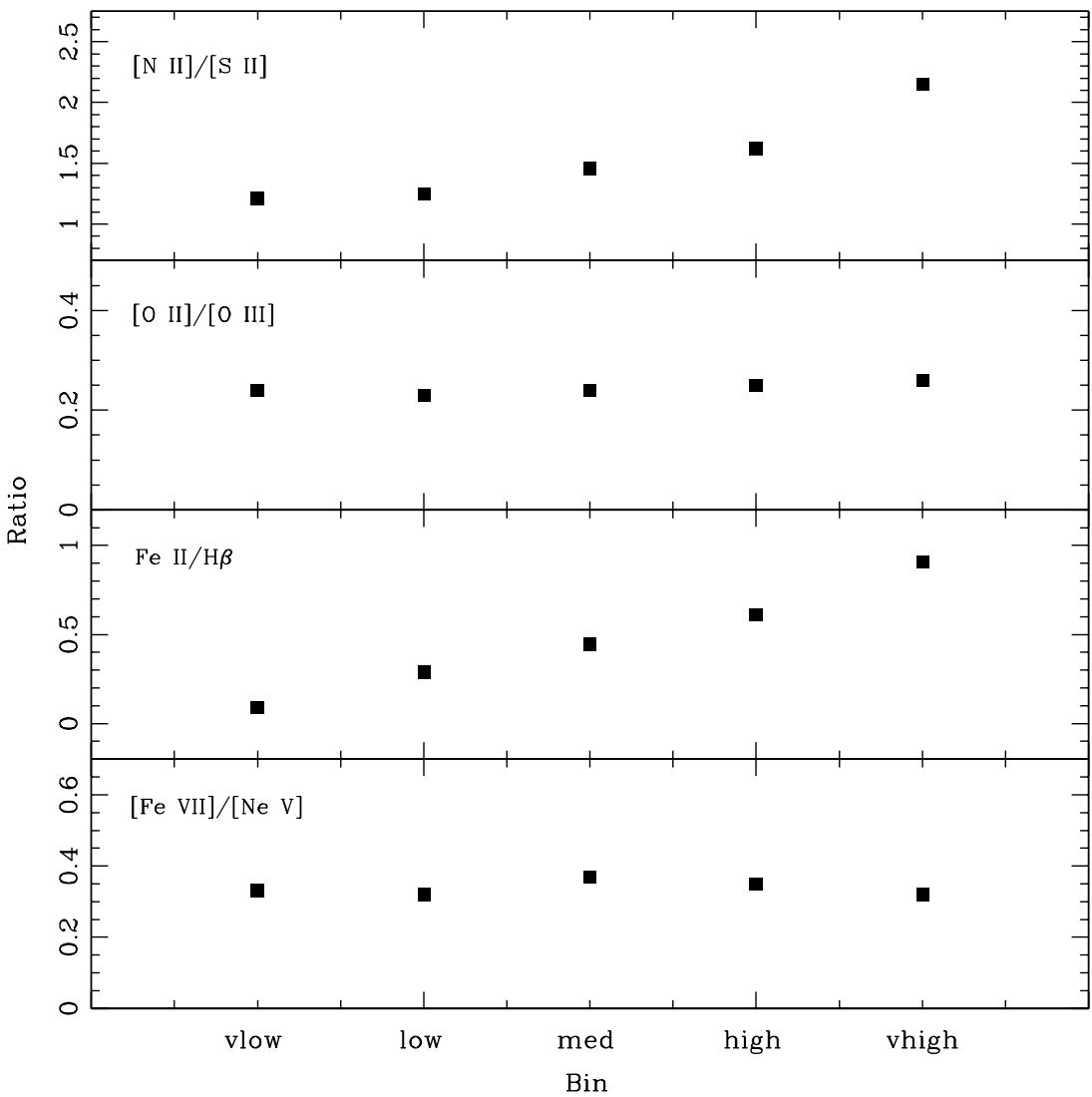

Figure 3. Narrow emission-line intensity ratios ratios for the five composite spectra. See the text for discussion including errors.

Table 1

Emission-Line Ratios for Composite Spectra

\begin{tabular}{lccccc}
\hline \hline \multicolumn{1}{c}{$\begin{array}{c}\text { Emission } \\
\text { Lines }\end{array}$} & vlow & low & $\begin{array}{c}\text { Intensity Ratio } \\
\text { med }\end{array}$ & high & vhigh \\
\hline$[\mathrm{N} \mathrm{II}] /\left[\mathrm{S} \mathrm{II}_{\mathrm{II}}\right.$ & 1.21 & 1.25 & 1.46 & 1.62 & 2.15 \\
{$[\mathrm{~N} \mathrm{II}] /\left[\mathrm{O}_{\mathrm{II}}\right]$} & 0.96 & 1.25 & 1.22 & 1.55 & 2.29 \\
{$\left[\mathrm{O}_{\mathrm{II}}\right] /\left[\mathrm{O}_{\mathrm{III}}\right]$} & 0.24 & 0.23 & 0.24 & 0.25 & 0.26 \\
{$[\mathrm{~S} \mathrm{II}] /[\mathrm{O} \mathrm{II}]$} & 0.80 & 0.95 & 0.83 & 0.95 & 1.07 \\
{$[\mathrm{Fe} \mathrm{VII}] /[\mathrm{Ne} \mathrm{v}]$} & 0.33 & 0.32 & 0.37 & 0.35 & 0.32 \\
{$[\mathrm{Ne} \mathrm{V}] /[\mathrm{Ne} \mathrm{III}]$} & 0.97 & 1.10 & 1.15 & 1.61 & 1.81 \\
$\mathrm{H} \alpha / \mathrm{H} \beta$ & 3.64 & 3.39 & 4.14 & 3.52 & 3.04 \\
{$[\mathrm{~S} \mathrm{II}](6720 / 4072)$} & $9.2:$ & $10.3:$ & $9.7:$ & $9.9:$ & $13.5:$ \\
{$[\mathrm{S} \mathrm{II}](6716 / 6731)$} & 1.19 & 1.05 & 1.07 & 1.10 & 1.08 \\
$\mathrm{Fe} \mathrm{II} / \mathrm{H} \beta$ & 0.11 & 0.29 & 0.44 & 0.61 & 0.91 \\
\hline
\end{tabular}

Notes. Intensity ratio for emission lines measured from composite spectra binned by broad Fe II strength. Values refer to the narrow emission lines except for the Balmer lines and Fe II. Intensities include both lines for the [O II] and [S II] doublets but only the stronger line for [N II], [O III], [Ne III], and [Ne V]. Colon indicates large uncertainty. See the text for discussion.

residual intensity or over-subtraction of [N $\mathrm{NI}]$. Error bars were estimated by determining [ $\mathrm{N}$ II] intensities giving a slight underor over-subtraction as judged by eye. This gave an uncertainty of about $\pm 12 \%$ for each composite.

Our results will be discussed in terms of trends of observed line ratios. For actual ionic abundances, collision strengths from Berrington et al. (2000) and Osterbrock \& Ferland (2006) lead to the relation

$$
n\left(\mathrm{Fe}^{+6}\right) / n\left(\mathrm{Ne}^{+4}\right)=0.91 I(\lambda 6087) / I(\lambda 3425)
$$

for an assumed $T=15,000 \mathrm{~K}$ based on photoionization models. Likewise, for the $\left[\mathrm{N}_{\mathrm{II}}\right] /\left[\mathrm{S}_{\mathrm{II}}\right]$ ratio we have

$$
n\left(\mathrm{~N}^{+}\right) / n\left(\mathrm{~S}^{+}\right)=6.0 I(\lambda \lambda 6584,6548) / I(\lambda \lambda 6716,6730)
$$

for an assumed $T=12,000 \mathrm{~K}$. These expressions give the ionic abundance ratios, if collisional de-excitation is unimportant.

\section{RESULTS FOR THE NARROW-LINE REGION}

\subsection{Iron}

Nussbaumer \& Osterbrock (1970) suggested that [Fe VII]/ $[\mathrm{Ne} \mathrm{v}]$ should be a good measure of the $\mathrm{Fe} / \mathrm{Ne}$ ratio, based on the similarity of the ionization potentials. There is no significant trend in $[\mathrm{Fe} \mathrm{VII}] /[\mathrm{Ne} \mathrm{v}]$ in our composite spectra. The Fe II $/ \mathrm{H} \beta$ ratio varies by a factor of 8 from the "very low" to "very high" composite. Taking neon to represent the $\alpha$-elements in general, we conclude that differences in $\mathrm{Fe}$ abundance, relative to the $\alpha$-elements, are not a significant cause of the observed range of Fe II emission strength, from a statistical point of view. In particular, overabundances of $\mathrm{Fe} / \mathrm{O}$ and $\mathrm{Fe} / \mathrm{Mg}$ of a factor of 2 to 10 , as motivated by chemical evolution models and attempts to fit the $\mathrm{Fe}$ II $/ \mathrm{Mg}$ II ratio with photoionization models (Wills et al. 1985; Hamann \& Ferland 1993), appear to be ruled out.

This conclusion is based on the assumption that the [Fe VII]/ $[\mathrm{Ne} \mathrm{v}]$ ratio is a faithful measure of $\mathrm{Fe} / \mathrm{Ne}$, and that $\mathrm{Fe}$ is not significantly depleted into grains in the [Fe VII] zone of the NLR. Ferguson et al. (1997b) and Nagao et al. (2003) conclude that refractory elements are not depleted in the coronal line region of the NLR, but they also find that [Fe VII] and [Ne v] do not come from the same place in "locally optimally emitting 
cloud" (LOC) models of the NLR. Here we assume that any ionization correction for $\mathrm{Fe}^{+6} / \mathrm{Ne}^{+4}$ in the NLR does not change systematically with the broad line Fe II intensity among our composites. We further assume that the abundances in the BLR are similar to those in the NLR. However, it is possible that intense star formation in the nucleus may give chemical enrichment on a spatial scale smaller than the NLR (see Hamann et al. 2007; Hamann \& Ferland 1999, and references therein).

\subsection{Ionization}

The composite spectra were originally constructed to assess the [Fe VII] strength in the NLR, but they also afford an opportunity to examine other narrow-line ratios for any systematic dependence on Fe II strength. One issue is the level of ionization of the gas. Table 1 and Figure 3 show that the $[\mathrm{O} \mathrm{II}] /[\mathrm{O} \mathrm{III}]$ ratio is closely similar for the various Fe II bins. There is a significant increase in [Ne v]/[Ne III] with increasing Fe II strength, reflecting a $\sim 25 \%$ decrease in the $[\mathrm{Ne} \mathrm{v}] \mathrm{EW}$ across the bins and a much larger decrease in the $\mathrm{EW}$ of [Ne III]. The narrow $\mathrm{He}$ II $\lambda 4686$ line to $[\mathrm{O}$ III] $\lambda 5007$ intensity ratio (not given in Table 1) shows a similar, though less continuous, increase from 0.06 to 0.13 across the five bins; this reflects a substantially constant EW of narrow He II together with a systematic decrease in the EW of $\lambda 5007$ from 25 to $12 \AA$ across the bins. The anticorrelation of Fe II and [O III] is well known (BG92). Although the constancy of [Fe VII]/[Ne v] could result from offsetting ionization and abundance trends, a straightforward interpretation is that the relative size of the zone containing $\mathrm{O}^{+2}$ and $\mathrm{Ne}^{+2}$ decreases with increasing Fe II without a major effect on the $\mathrm{Fe}^{+6} / \mathrm{Ne}^{+4}$ ionization correction.

\subsection{Reddening}

The $[\mathrm{S} \mathrm{II}] /[\mathrm{O} \mathrm{II}]$ ratio shows an increase of about $30 \%$ with increasing Fe II strength. This may be an indication of a modest increase in reddening of the NLR with increasing Fe II. The [S II] $\mathrm{I}(\lambda 6720) / \mathrm{I}(\lambda 4072)$ ratio is quite uncertain but is consistent with a reddening trend of this magnitude. This trend is not evident in the broad $\mathrm{I}(\mathrm{H} \alpha) / \mathrm{I}(\mathrm{H} \beta)$ intensity ratio.

\subsection{Nitrogen}

The $[\mathrm{N} \mathrm{II}] /[\mathrm{S}$ II $]$ ratio shows a systematic increase by a factor of 2 from the very low to the very high Fe II bins. This ratio is insensitive to reddening and electron temperature. The $\mathrm{N}^{+}$and $\mathrm{S}^{+}$ions have similar ionization potentials and occupy similar zones of the nebular ionization structure. This and the constancy of $[\mathrm{OII}] /[\mathrm{O} \mathrm{III}]$ suggests that the trend in $[\mathrm{N} \mathrm{II}] /[\mathrm{S} \mathrm{II}]$ is not a result of ionization of the NLR. The trend of increasing $[\mathrm{NII}]$ strength is evident in the $[\mathrm{NII}] /[\mathrm{O}$ II $]$ and $[\mathrm{N}$ II $] /[\mathrm{O}$ III $]$ ratios as well. These results imply a real trend in the $\mathrm{N} / \mathrm{S}$ and $\mathrm{N} / \mathrm{O}$ chemical abundance ratios with increasing Fe II strength, amounting to a factor of 2 from the "very low" to the "very high" Fe II strengths. Nitrogen is largely a secondary nucleosynthetic product, so that $\mathrm{N} / \mathrm{O}$ increases with $\mathrm{O} / \mathrm{H}$. The $\mathrm{H}$ iI region results of van Zee et al. (1998) show N/O increasing almost linearly with $\mathrm{O} / \mathrm{H}$ above $12+\log \mathrm{O} / \mathrm{H}=8.5$. Assuming that $\mathrm{O} / \mathrm{H}$ is in this range in the AGN studied here, then the trend of $\mathrm{N} / \mathrm{O}$ with Fe II implies an increase of a factor of 2 in $\mathrm{O} / \mathrm{H}$. Although the chemical evolution of AGN host galaxies may be complicated (Hamann \& Ferland 1999; Hamann et al. 2002; Netzer \& Trakhtenbrot 2007), it may be reasonable to assume that $\mathrm{Fe} / \mathrm{H}$ varies roughly with $\mathrm{O} / \mathrm{H}$ for the modest redshifts considered here. In this case, the $\mathrm{Fe} / \mathrm{H}$ abundance ratio may contribute roughly a factor of 2 to the range in Fe II strength from our "very low" to "very high" bins. This conclusion is consistent with the work of Netzer \& Trakhtenbrot (2007), who find an increase of Fe II strength with Eddington ratio $L / L_{\mathrm{Ed}}$ in a large sample of SDSS quasars. Combining this with indications of an increasing N/C with $L / L_{\mathrm{Ed}}$ in the BLR (Scott et al. 2004; Hamann et al. 2002), they argue that the overall metallicity in QSOs increases with Fe II strength. However, our results indicate that this makes only a modest contribution to the full range of optical Fe II strength in AGN. Note, however, that our QSO sample is at low redshift, whereas much of the interest in iron abundances in QSOs has focused on high redshifts.

\section{Fe II STRENGTH AND X-RAY HEATING}

It is widely assumed that the Fe II emission is largely powered by the soft X-ray portion of the ionizing spectrum, given that photoionization is indeed the primary excitation mechanism. The harder photons in the ionizing continuum create an extensive warm, partially ionized zone where Fe II and other low ions are subject to collisional excitation and in some cases other excitation mechanisms (see Kwan \& Krolik 1981; Ferland et al. 2009, and references therein). Does the relative strength of the X-ray continuum drive the range of Fe II strength observed in AGN? The following considerations suggest that it does not.

1. A number of empirical studies have examined correlations of Fe II strength with the X-ray slope or with the X-ray/ optical ratio (Sulentic et al. 2000, and references therein). Lawrence et al. (1997) studied a sample of AGN with extreme values of $R_{\mathrm{FeII}} \equiv \mathrm{I}(\lambda 4570) / \mathrm{I}(\mathrm{H} \beta)$. The strong Fe II emitters have X-ray properties, including $\alpha_{\mathrm{ox}}$, similar to the weak Fe II objects. In particular, the prototype strong Fe II object, I Zw 1 , has $\alpha_{\text {ox }}=-1.4$, an entirely typical value. Combining their data with the complete sample of Laor et al. (1997), Lawrence et al. found little correlation between $\alpha_{\mathrm{ox}}$ and $R_{\mathrm{FeII}}$, and only an ambiguous correlation with $\alpha_{\mathrm{x}}$. Indeed, Figure 3 of Lawrence et al. shows a slight trend in the sense of strong optical Fe II for weak X-ray luminosity (steep $\alpha_{\mathrm{ox}}$ ). They did find a significant correlation with the X-ray to IR slope $\alpha_{\mathrm{ix}}$ in the sense of stronger infrared for stronger Fe II. Using composite spectra for X-ray bright and X-ray faint QSOs, Green (1998) found that UV Fe II was stronger whereas optical Fe II was weaker for $\mathrm{X}$-ray bright objects. However, the differences were small compared to the full range of Fe II strength among individual AGN. Leighly et al. (2007) find a weak X-ray continuum but strong Fe II emission in PHL 1811, and discuss other similar examples. None of these results gives support for the idea that stronger X-ray continuum drives stronger Fe II for AGN as a class. Ferland \& Persson (1989) reached a similar conclusion regarding the Ca II emission from AGNs, which also comes from the partially ionized zone.

2. In order to explore the expected response of the Fe II emission to differences in X-ray luminosity (and other parameters) we have computed a set of models of the BLR using version 07.02.00 of the photoionization code Cloudy, most recently described by Ferland et al. (1998). As a reference model, following Ferland et al. (2009), we used solar abundances, an ionizing flux $\phi=10^{19} \mathrm{~cm}^{-2} \mathrm{~s}^{-1}$, and a gas density $N=10^{10} \mathrm{~cm}^{-3}$, giving an ionization parameter $U \equiv \phi / N c=10^{-1.5}$. The internal turbulent velocity was $u_{\text {turb }}=100 \mathrm{kms}^{-1}$, and the stopping column 
Table 2

Photoionization Model Results

\begin{tabular}{lcccc}
\hline \hline \multicolumn{1}{c}{ Model } & 1 & 2 & 3 & 4 \\
$\quad \alpha_{\text {Ox }}$ & -1.4 & -2.0 & -1.4 & -1.4 \\
Fe Depletion & 0.0 & 0.0 & -1.5 & -1.5 \\
Other Depletion & 0.0 & 0.0 & 0.0 & -1.5 \\
\hline \multicolumn{5}{c}{$I^{\prime} I_{\mathrm{H} \beta}$} \\
\hline 1500 & 6.2 & 7.5 & 0.207 & 1.59 \\
2500 & 17.8 & 21.0 & 5.1 & 3.9 \\
5000 & 0.55 & 0.58 & 0.029 & 0.024 \\
7000 & 0.069 & 0.081 & 0.0059 & 0.0045 \\
10000 & 0.42 & 0.44 & 0.030 & 0.024 \\
Mg II & 3.4 & 3.2 & 2.8 & 0.20 \\
Si III $]$ & 1.30 & 1.46 & 1.19 & 0.037 \\
Si IV & 1.56 & 1.75 & 1.42 & 0.104 \\
H $\alpha$ & 4.6 & 4.6 & 4.5 & 4.6 \\
Ly $\alpha$ & 28.1 & 32.4 & 25.8 & 21.6 \\
C III] & 1.69 & 1.78 & 1.54 & 1.45 \\
C IV & 13.8 & 13.2 & 12.6 & 11.1 \\
\hline
\end{tabular}

Notes. Results of Cloudy photoionization models. Logarithmic depletion relative to hydrogen is given for iron and for other refractory elements. See the text for discussion.

density was $10^{23} \mathrm{~cm}^{-2}$, in order to include an extensive partially ionized zone. The ionizing continuum was the sum of (1) a UV component $L_{v} \propto v^{-0.5} \exp \left(-h v / k T_{\text {cut }}\right)$ with $T_{\text {cut }}=10^{5.7} \mathrm{~K}$ to represent the big blue bump, and (2) an X-ray power law $L_{v} \propto v^{-1}$. The continuum had a low frequency exponential cutoff below 0.01 Ryd. The models used the full treatment of the Fe II ion (371 levels) and were iterated to convergence of the diffuse radiation field. The ratio of the X-ray to the UV component is controlled by the parameter $\alpha_{\mathrm{ox}}$. For the reference model (Model 1), we used $\alpha_{\mathrm{ox}}=-1.4$, a typical observed value (Lawrence et al. 1997). This model has not been optimized to fit a typical AGN emission-line spectrum, but simply serves as a reference point for exploring the effect on Fe II of changing various model parameters. A simultaneous fit to AGN emission-line spectra requires a combination of photoionized clouds with a range of physical conditions (Baldwin et al. 1995).

Table 2 gives line intensities from the Cloudy models. The $\mathrm{H} \alpha$, [C III], $\mathrm{CIV}_{\mathrm{IV}}$, and $\mathrm{Mg}$ II intensities are reasonable. Ly $\alpha$ is stronger than observed relative to $\mathrm{H} \beta$, a familiar problem with photoionization models (e.g., Kwan \& Krolik 1981). The Cloudy output gives Fe II intensities summed over broad wavelength bands at 1000-2000, 2000-3000, 4000-6000, 6000-7800, and 7800-30,000 $\AA$. Note that the $\lambda 2500$ band includes the broad UV Fe II bump, and the $\lambda 5000$ band contains the prominent optical Fe II blends at $\lambda 4570$ and $\lambda 5250$. Model 1 underpredicts the Fe II intensity seen in strong Fe II objects, a common problem with photoionization models as mentioned above.

In Model 2, we used $\alpha_{\text {ox }}=-2.0$ to explore the effect of weaker X-rays while remaining within the span of frequently observed values of $\alpha_{\text {ox }}$ (Lawrence et al. 1997). The Fe II intensity (relative to $\mathrm{H} \beta$ ) actually increased slightly in the X-ray weak model. We also computed an alternative pair of models with $\phi=10^{18} \mathrm{~cm}^{-2} \mathrm{~s}^{-1}, N=10^{9} \mathrm{~cm}^{-3}, T_{\text {cut }}=10^{5.3} \mathrm{~K}$, and no turbulence. In this case, the Fe II $\lambda 2500$ blend decreased by 0.16 dex and the $\lambda 5000$ band by 0.20 dex in going from $\alpha_{\mathrm{ox}}=-1.4$ to -2.0 . Even in this case, the degree of change is insufficient to give the full observed range of Fe II strength. An extreme systematic variation of $\alpha_{\mathrm{ox}}$ with Fe II would be required, for which the observations give little support.

\section{Fe II STRENGTH AND IRON DEPLETION INTO GRAINS}

The above results indicate that changes in the total elemental abundance of Fe and the X-ray luminosity do not cause the wide observed range of Fe II emission strength among AGNs. We propose instead that Fe II strength in AGNs largely results from differing degrees of depletion of the gas-phase abundance of iron into grains in the relevant portions of the BLR. Gas-phase depletions of refractory elements in the interstellar medium can be severe. Iron is depleted by up to 2 orders of magnitude in the interstellar medium and in ionized nebulae (see discussion below). Such a degree of depletion, if present in AGNs with weak Fe II but not those with strong Fe II, can account for much of the observed range of Fe II strength in AGNs.

There has been considerable discussion of refractory element depletions in the NLRs of AGNs (Ferguson et al. 1997a). Gaskell et al. (1981) considered the question of refractory element depletions in the BLR and concluded that depletions of $\mathrm{Si}$, $\mathrm{Mg}$, and Fe as severe as those in the ISM did not occur. This conclusion was based mostly on the Mg II, Si III, and Si IV lines, and the intensity of Fe II in strong Fe II objects. Here we suggest that the degree of depletion of iron varies from object to object, and can be severe in objects with weak Fe II. In a study of [O I] and Ca II emission from AGNs, Matsuoka et al. (2008) note that depletion of Ca II might help to reconcile predicted and observed intensities and mention the possibility that depletions may affect the Fe II lines. Ferland \& Persson (1989) note that dust mixed with the BLR gas could help to explain low observed values of the $\mathrm{Ca}$ II $\mathrm{H}$ and $\mathrm{K}$ emission lines, relative to the infrared triplet. As discussed below, the radius of the BLR is interestingly close to the dust sublimation radius; and for typical parameters, grains may exist in the partially ionized zone of the BLR clouds but not in the highly ionized surface layers.

The present proposal does not address the long-standing question of how to explain the large Fe II strength observed in many AGNs (Wills et al. 1985; Bruhweiler \& Verner 2008, and references therein). Rather, it serves to separate the question of Fe II strength into two parts: (1) why is Fe II so strong in some objects, and (2) why is it so weak in others? We do not attempt to resolve the first question in this paper, except to note that under the present proposal, the Fe II physical excitation mechanism is freed from the requirement of explaining by itself the wide range of observed Fe II strengths.

\subsection{Photoionization Models}

In order to explore the dependence of Fe II strength on gas-phase $\mathrm{Fe}$ abundance we have computed two additional Cloudy models (see Table 2). Model 3 is otherwise identical to Model 1 but has a depletion of iron by $1.5 \mathrm{dex}$. The $\lambda 2500$ feature decreased in intensity by $0.66 \mathrm{dex}$, and the $\lambda 5000$ feature by 1.36 dex, corresponding to $(\mathrm{Fe} / \mathrm{H})^{0.44}$ and $(\mathrm{Fe} / \mathrm{H})^{0.91}$, respectively. In the $N=10^{9} \mathrm{~cm}^{-3}$ model described above, a 1.5 dex depletion of Fe abundance reduced the $\lambda 2500$ and $\lambda 5000$ features by 0.82 dex and 1.37 dex, respectively. Thus, the optical Fe II intensity varied almost linearly with the gasphase iron abundance, and the UV Fe II less strongly. These results are consistent with previous photoionization studies. Verner et al. (2003) and Baldwin et al. (2004) found that the Fe II intensity varies approximately as $(\mathrm{Fe} / \mathrm{H})^{0.8}$ for the optical 
lines and as $(\mathrm{Fe} / \mathrm{H})^{0.4}$ for the UV bump. Some of these models assume different physical parameters for the BLR from ours, and the Verner et al. (2003) models use an 830 level $\mathrm{Fe}^{+}$model atom as opposed to 371 levels in our models and those of Baldwin et al. (2004).

Other refractory elements may be depleted where iron is. In Model 4, the abundances of $\mathrm{Al}, \mathrm{Mg}, \mathrm{Si}, \mathrm{Ca}$, and $\mathrm{Fe}$ were all depleted by 1.5 dex. Table 2 shows that the multiplets Mg II $\lambda 2800$, Si III $\lambda 1892$, and Si IV $\lambda 1400$ decreased roughly in proportion to the gas-phase abundance. The behavior of the Fe II multiplets in Model 4 is similar to the case in which only $\mathrm{Fe}$ was depleted.

Different refractory elements have different depletions in the ISM, and different grain compositions may be more or less easily destroyed in the AGN environment. This may lead to useful diagnostics for grain destruction and gas-phase depletions. Delgado Inglada et al. (2009) summarize published depletion factors in planetary nebulae: $1 / 6$ to $1 / 300$ for $\mathrm{Ca}, 1 / 2$ to $1 / 350$ for $\mathrm{Al}, 1 / 3$ to $1 / 300$ for $\mathrm{Fe}$, near solar to $1 / 10$ for $\mathrm{Mg}$, and near solar to $1 / 20$ for Si. Silicon appears to be depleted by a lesser factor than iron in HiI regions. Garnett et al. (1995) found Si depletions of only -0.1 to -0.6 dex in extragalactic $\mathrm{H}$ II regions, significantly less than in dense interstellar clouds. If $\mathrm{Si}$ is more easily restored to the gas phase in $\mathrm{H}$ II regions, the same may be true in AGNs, allowing objects with weak Fe II to have normal intensities of Si III and Si IV.

\subsection{Geometrical Considerations}

What might cause widely differing degrees of depletion of iron, and possibly other refractory elements, among AGNs? From considerations of BLR covering factor, Lyman continuum absorption, line widths, and reverberation mapping, Gaskell (2009) argues that the BLR is the inward extension of the dusty torus, with an inflow velocity a substantial fraction of the orbital velocity. Such a picture lends itself to the idea of refractory element depletions in the low ionization zone. As material flows inward through the dusty torus, its equilibrium temperature rises as it experiences a stronger radiation field, eventually reaching the sublimation temperature of $\sim 1500 \mathrm{~K}$. The exact sublimation radius depends on grain size and composition (Laor \& Draine 1993), but there will be a point where the refractory elements are substantially restored to the gaseous phase. This sublimation radius is given by Laor \& Draine (1993) as $R_{\mathrm{d}}=(0.2 \mathrm{pc}) L_{46}$, where $L_{46}$ is the AGN luminosity in units of $10^{46} \mathrm{ergs}^{-1}$. If this happens before the material reaches the main region of low ionization line emission, then strong emission in Fe II and other lines of refractory elements will occur. On the other hand, if the dust survives through most of the low ionization zone, then these emission lines will be weak, because of the lack of emitting ions and because of attenuation of the ionizing radiation and line emission by the dust itself.

Netzer \& Laor (1993) suggested that the survival of dust outside the sublimation radius leads to extinction of the ionizing continuum and suppression of line emission, setting a natural limit to radius of the BLR (see also Laor 2007). Infrared variability studies give a radius of the dusty torus just outside the BLR (Suganuma et al. 2006), supporting this picture. Here we suggest that the relationship between the sublimation radius and the BLR outer boundary differs from object to object. Critical to this picture is the actual radius of Fe II emission relative to the dust sublimation radius. Reverberation studies and line widths indicate that the lower ionization lines often come from larger radii in the BLR (Peterson 1997; Sulentic et al. 2000).
This may result from some combination of actual ionization stratification and line emissivity effects as considered in the LOC model (Baldwin et al. 1995). There have been few successful reverberation studies of the Fe II emitting radius in AGNs. For the Seyfert galaxy, NGC 5548, Maoz (1993) found a similar time lag for the UV Fe II lines as for Ly $\alpha$. In a reverberation study of Ark 120, which has strong optical Fe II, Kuehn et al. (2008) found that the lag for the optical Fe II lines was ill defined but may be around 300 days, larger than for $\mathrm{H} \beta$. They estimate a dust sublimation radius in this object of $\sim 460$ light days, and conclude that within the uncertainties "it is plausible that the optical Fe II emission is produced at or just inside the dust sublimation radius." In a study of line profiles of AGNs in SDSS, $\mathrm{Hu}$ et al. (2008) conclude that the Fe II emission comes from an inflowing zone in the outer part of the BLR. Taken together, these studies are consistent with Fe II emission from gas entering the BLR from the dusty torus.

A refinement of this picture takes account of the attenuation of the ionizing radiation field with depth within the ionized cloud or ionized surface layer of the disk or torus. Reverberation measurements of the BLR radius (often involving the $\mathrm{H} \beta$ line) typically give a radius smaller than the dust sublimation radius by a factor of order 2 , based on radii and luminosities given by Bentz et al. (2009). Thus, refractory grains should not survive at the illuminated face of a BLR cloud. However, the radiation field diminishes with depth in the cloud, allowing dust to survive at large depths. As the dusty gas flows from deep in the torus toward the irradiated surface, the ambient radiation field intensifies and the grain temperature rises, reaching the sublimation point at some depth in the ionization structure. Grain equilibrium temperatures calculated by the Cloudy program support this picture. In the reference model (Model 1) described above, the temperature of silicate grains with a radius of 0.094 microns is $680 \mathrm{~K}$ at the maximum column depth of $10^{23} \mathrm{~cm}^{-2}$. This allows grains to survive at this depth, even though the incident flux at the cloud face would easily evaporate refractory grains. The grain temperature rises with decreasing depth, reaching the sublimation temperature of $1500 \mathrm{~K}$ at a column density of $10^{21.63} \mathrm{~cm}^{-2}$. This is well below the "Strömgren depth" of $10^{21.23} \mathrm{~cm}^{-2}$, where hydrogren is $50 \%$ ionized. The grain temperature as calculated by Cloudy would be $2000 \mathrm{~K}$ at this latter depth, and $3100 \mathrm{~K}$ at the cloud surface. Reference to Figure 3 of Ferland et al. (2009) shows that in their model (similar to our Model 1), most of the optical Fe II emission occurs below the sublimation depth of $10^{21.63} \mathrm{~cm}^{-2}$, whereas most of the ultraviolet Fe II emission occurs at shallower depths. Thus, evaporation of the grains occurs at a depth giving severe reduction of the optical but not the ultraviolet Fe II emission. Dust will also not affect the emission from the highly ionized gas above the Strömgren depth, such as [Si III] and Si IV; and weak optical Fe II could accompany a normal ratio of the ultraviolet Fe II to Mg II lines. The exact sublimation depth depends on grain size and composition, but the qualitative pattern remains for a range of compositions and sizes included in the Cloudy output. For different AGN with different parameters, the sublimation point will occur at different depths in the ionization structure. Gaskell et al. (2007) have also discussed the affect of attenuation of the AGN continuum in the BLR on the radius of dust sublimation.

An alternative geometry for the BLR involves a radiatively driven wind from the accretion disk (Murray \& Chiang 1998). In this picture, one issue is whether grains can survive in the inflowing material to the radius where it is expelled in the wind. 
Standard accretion disk physics (Peterson 1997) gives for the disk effective temperature due to internal viscous dissipation

$$
T_{\text {eff }}=\left(10^{3.12} \mathrm{~K}\right)\left(\dot{M} / \dot{M}_{\mathrm{E}}\right)^{1 / 4} M_{8}^{-1 / 4} v_{3000}^{3 / 2},
$$

where $\dot{M} / \dot{M}_{\mathrm{E}}$ is the accretion rate relative to that giving the Eddington luminosity, $M_{8}$ is the black hole mass in units of $10^{8} M_{\odot}$, and $v_{3000}$ is the orbital velocity at the radius of interest in units of $3000 \mathrm{~km} \mathrm{~s}^{-1}$. Thus, the sublimation temperature for refractory grains is reached at orbital velocities appropriate for the broad emission lines. This may be consistent with differing degrees of grain evaporation in different objects. (The mid-plane temperature of the disk will be higher.) The above expression qualitatively agrees with the observed trend of stronger optical Fe II with increasing Eddington ratio, but the dependence on black hole mass may be problematic. Moreover, it does not give such a natural $L^{0.5}$ dependence for the BLR radius as does the dust sublimation model of Netzer \& Laor (1993). Note that in the disk-wind picture, the energy for the line emission still comes from photoionization by the central continuum; locally produced energy is insufficient at this shallow depth in the gravitational potential. Sublimation at an intermediate depth in the ionization structure, as discussed above, could also occur in the disk-wind model.

\subsection{Turbulent Velocity and Column Density}

Local turbulence substantially affects the Fe II spectrum in photoionization models by facilitating continuum and line-line fluorescence. Increasing the turbulence can increase the Fe II strength and give better agreement between the predicted shape of the Fe II blends and observation (Baldwin et al. 2004; Bruhweiler \& Verner 2008, and references therein). Bruhweiler \& Verner (2008) find a factor of 2 increase in the UV Fe II strength relative to $\mathrm{Mg}$ II as the turbulence increases from 5 to $50 \mathrm{~km} \mathrm{~s}^{-1}$ (their Table 2). When we decreased $v_{\text {turb }}$ from 100 $\mathrm{km} \mathrm{s}^{-1}$ to zero in Model 1 , the $\lambda 2500 \mathrm{Fe}$ II band decreased by a factor of 10 , and the $\lambda 5000$ band decreased by a factor of 2 . If these results are representative, they suggest that differing amounts of turbulence could make a substantial contribution to the range of strength of Fe II in AGNs. On the other hand, turbulence still appears to be inadequate to give the full observed range in optical Fe II. Moreover, Baldwin et al. (2004) argue that substantial turbulence is required to fit the detailed shape of the UV Fe II feature, so that there may be limited freedom to vary the turbulence. A useful test may be to examine the observed shape of the UV and optical Fe II blends as a function of optical Fe II strength, for comparison with photoionization models that vary either the turbulence or the gas-phase abundance of $\mathrm{Fe}$.

The column density of the emitting clouds also has an important effect on the Fe II emission. Ferland et al. (2009) illustrate the increase in $\mathrm{Fe} \mathrm{II} / \mathrm{H} \beta$ with increasing column density. They find that the minimum column density is $\sim 10^{23} \mathrm{~cm}^{-2}$ for gravity to overpower radiation pressure and allow infall of clouds as found by Hu et al. (2008). The UV Fe II lines show little change above this column density, but the optical Fe II increases a factor $\sim 0.5$ dex from $\sim 10^{23} \mathrm{~cm}^{-2}$ to $\sim 10^{25} \mathrm{~cm}^{-2}$. Using arguments based on virial determinations of the black hole mass in AGNs, Netzer (2008) also concludes that the column densities must substantially exceed $\sim 10^{23} \mathrm{~cm}^{-2}$ to avoid excessive effects of radiation pressure on the orbital velocities of the BLR clouds. Thus, there may be limited freedom to vary the column density in order to produce the wide range of optical Fe II strength observed.
The relative behavior of the optical and UV Fe II bands may provide clues to the predominant cause of the range of Fe II strength. In our models, the UV and optical Fe II both increase with $\mathrm{Fe}$ abundance, albeit more weakly for the UV blends. However, increasing the microturbulence increased the UV Fe II by a greater factor that the optical Fe II; and increasing the column density beyond $\sim 10^{23} \mathrm{~cm}^{-2}$ mainly increases the optical Fe II, as noted above. Shang et al. (2007) give optical and Fe II strengths for a sample of AGN. Their results show a much greater range in the $\mathrm{EW}$ of the optical $\mathrm{Fe}$ II bands than in the UV bands, and a weak anti-correlation between the optical and UV (see also Wills et al. 1985). The fact that the optical Fe II shows a greater range of intensity than the UV Fe II may favor an explanation other than microturbulence. One complication is whether the optical and UV lines originate at substantially different radii in the BLR, as suggested by some reverberation and line-width studies (e.g., Maoz 1993; Hu et al. 2008). Ferland et al. (2009) suggest that the observed optical Fe II may be strongly affected by radiation escaping from the shielded face of the photoionized clouds. The observational and theoretical situation for Fe II is complex, and further work will be needed to devise definitive tests of the role of chemical abundances and physical conditions.

\section{CONCLUSION}

We have used composite SDSS spectra of AGNs to examine the behavior of the narrow emission lines as a function of Fe II strength. The [Fe VII] line shows only a weak increase with increasing Fe II strength, indicating that the iron abundance contributes little to the wide range of Fe II strength in AGNs. There is, however, a significant increase in the N/O abundance ratio with Fe II strength, which suggests an increase in overall metallicity. There is little change in the level of ionization in the NLR as a function of Fe II strength. This, together with results of photoionization models, suggests that differences in the shape of the ionizing continuum, specifically the soft X-ray luminosity, are not the main drivers of the Fe II strength. We propose that differences in the degree of depletion of $\mathrm{Fe}$ into grains in the low ionization portion of the BLR are largely responsible for the weakness of Fe II in some AGNs, while it is strong in others. This picture is consistent with the approximate coincidence of the BLR radius and the dust sublimation radius, with indications that the BLR consists of material flowing inward from the dusty torus toward the central black hole, and with the variation of grain temperature with depth in the ionization structure of the BLR gas.

The strength of Fe II emission is a major component of the set of correlations known as "Eigenvector 1" (EV1) discussed by BG92. These include weak [O III] associated with strong Fe II and narrower widths of the broad $\mathrm{H} \beta$ line. Radio-loud AGNs tend to have strong [O III] and weak Fe II. These trends have been the subject of many studies, but a good physical understanding of their origin remains lacking. BG92 suggested that high column densities in the BLR enhance Fe II while diminishing the ionizing radiation reaching the NLR. Ludwig et al. (2009), in a spectral principal components analysis of AGNs in SDSS, found the interpretation of the eigenvectors to be complicated. They argued that covering factor of the NLR was the likely cause of the range in [O III] strength. Ferland et al. (2009) suggest that the higher column densities required for infall in more luminous AGNs can help to explain the correlation of Fe II strength with $L / L_{\mathrm{Ed}}$. 
The interpretation of Fe II strength in terms of dust depletion opens many questions for investigation. Can support for this picture be found in the line intensities of other refractory elements? Does the Fe II emitting radius bear a different relationship to the dust sublimation radius for strong and weak Fe II emitters? What are the implications for the infrared emission of AGNs? What underlying causes lead to the correlations between Fe II strength and other properties such as [O III] strength, radio emission, and Eddington ratio? This paper does not offer answers to these larger questions, but the explanation of Fe II strength in terms of gas-phase depletions gives a new context in which to address them.

We thank Mark Botorff, Gary Ferland, Martin Gaskell, Richard Green, Fred Hamann, Ari Laor, Hagai Netzer, and Bev Wills for helpful discussions and comments on the manuscript, and Alyx Stevens for assistance. G.S. acknowledges support from the Jane and Roland Blumberg Centennial Professorship in Astronomy at the University of Texas at Austin. We thank Karl Gebhardt for the composite spectrum program and Erin Bonning for the emission-line subtraction program used for the nitrogen line measurements. Funding for the Sloan Digital Sky Survey (SDSS) has been provided by the Alfred P. Sloan Foundation, the Participating Institutions, the National Aeronautics and Space Administration, the National Science Foundation, the U.S. Department of Energy, the Japanese Monbukagakusho, and the Max Planck Society. The SDSS is managed by the Astrophysical Research Consortium (ARC) for the Participating Institutions. The Participating Institutions are The University of Chicago, Fermilab, the Institute for Advanced Study, the Japan Participation Group, The Johns Hopkins University, the Korean Scientist Group, Los Alamos National Laboratory, the Max-Planck-Institute for Astronomy (MPIA), the MaxPlanck-Institute for Astrophysics (MPA), New Mexico State University, University of Pittsburgh, University of Portsmouth, Princeton University, the United States Naval Observatory, and the University of Washington.

\section{REFERENCES}

Antonucci, R. R. J., \& Miller, J. S. 1985, ApJ, 297, 621

Baldwin, J., Ferland, G., Korista, K., \& Verner, D. 1995, ApJL, 455, L119

Baldwin, J. A., Ferland, G. J., Korista, K. T., Hamann, F., \& LaCluyzé, A. 2004, ApJ, 615, 610

Bentz, M. C., Peterson, B. M., Netzer, H., Pogge, R. W., \& Vestergaard, M. 2009, ApJ, 697, 160

Berrington, K. A., Nakazaki, S., \& Norrington, P. H. 2000, A\&AS, 142, 313

Boroson, T. A. 2002, ApJ, 565, 78

Boroson, T. A., \& Green, R. F. 1992, ApJS, 80, 109 (BG92)

Bruhweiler, F., \& Verner, E. 2008, ApJ, 675, 83

Collin, S., Hameury, J.-M., \& Joly, M. 1988, A\&A, 205, 19

Collin, S., \& Joly, M. 2000, New Astron., 44, 531

Delgado Inglada, G., Rodríquez, M., Mampaso, A., \& Vironen, K. 2009, ApJ, 693,1335
Ferguson, J. W., Korista, K. T., Baldwin, J. A., \& Ferland, G. J. 1997a, ApJ, 487, 122

Ferguson, J. W., Korista, K. T., \& Ferland, G. J. 1997b, ApJS, 110, 287

Ferland, G. J., Korista, K. T., Verner, D. A., Ferguson, J. W., Kingdon, J. B., \& Verner, E. M. 1998, PASP, 110, 761

Ferland, G. J., \& Persson, S. E. 1989, ApJ, 347, 656

Ferland, G. J., Hu, C., Wang, J.-M., Baldwin, J. A., Porter, R. L., van Hoof, P. A. M., \& Williams, R. J. R. 2009, ApJ, 707, L82

Garnett, D. R., Dufour, R. J., Peimbert, M., Torres-Peimbert, S., Shields, G. A., Skillman, E. D., Terlevich, E., \& Terlevich, R. J. 1995, ApJ, 449, L77

Gaskell, M. 2009, New Astron., 53, 140

Gaskell, C. M., Klimek, E. S., \& Nazarova, L. S. 2007, arXiv:0711.1025

Gaskell, C. M., Shields, G. A., \& Wampler, 1981, ApJ, 249, 443

Green, P. J. 1998, ApJ, 498, 170

Hamann, F., \& Ferland, G. 1999, ARA\&A, 37, 487

Hamann, F., \& Ferland, G. J. 1993, ApJ, 418, 11

Hamann, F., Korista, K. T., Ferland, G. J., Warner, C., \& Baldwin, J. 2002, ApJ, 564,592

Hamann, F., Warner, C., Dietrich, M., \& Ferland, G. 2007, in ASP Conf. Ser. 373 The Central Engine of Active Galactic Nuclei, ed. Luis C. Ho \& Jian-Min Wang (San Francisco, CA: ASP), 653

Hu, C., Wang, J.-M., Ho, L. C., Chen, Y.-M., Zhang, H.-T., Bian, W.-H., \& Xue, S.-J. 2008, ApJ, 687, 78

Kovačević, J., Popović, L. C., \& Dimitrijević, M. S. 2010, ApJS, 189, 15

Kuehn, C. A., Baldwin, J. A., Peterson, B. M., \& Korista, K. T. 2008, ApJ, 673, 69

Kwan, J., \& Krolik, J. H. 1981, ApJ, 250, 478

Laor, A. 2007, in ASP Conf. Ser. 373, The Central Engine of Active Galactic Nuclei, ed. Luis C. Ho \& Jian-Min Wang (San Francisco, CA: ASP), 383

Laor, A., \& Draine, B. T. 1993, ApJ, 402, 441

Laor, A., Fiore, F., Elvis, M., Wilkes, B. J., \& McDowell, J. C. 1997, ApJ, 477, 93

Lawrence, A., Elvis, M., Wilkes, B J., McHardy, I., \& Brandt, N. 1997, MNRAS, 285,879

Leighly, K. M., Halpern, J. P., Jenkins, E. B., \& Casebeer, D. 2007, ApJS, 173,

Ludwig, R. R., Wills, B., Greene, J. E., \& Robinson, E. L. 2009, ApJ, 706, 995 Maoz, D., et al. 1993, ApJ, 404, 576

Marziani, P., Zamanov, R. K., Sulentic, J. W., \& Calvani, M. 2003, MNRAS, 435, 1133

Matsuoka, Y., Kawara, K., \& Oyabu, S. 2008, ApJ, 673, 62

Murray, N., \& Chiang, J. 1998, ApJ, 494, 125

Nagao, T., Murayama, T., Shioya, Y., \& Taniguchi, Y. 2003, AJ, 125, 1729

Netzer, H. 2008, ApJ, 695, 793

Netzer, H., \& Laor, A. 1993, ApJ, 404, L51

Netzer, H., \& Trakhtenbrot, B. 2007, ApJ, 654, 754

Nussbaumer, H., \& Osterbrock, D. E. 1970, ApJ, 161, 811

Osterbrock, D. E., \& Ferland, 2006, Astrophysics of Gaseous Nebulae and Active Galactic Nuclei (2nd ed.; Sausalito, CA: Univ. Science Books)

Peterson, B. 1997, An Introduction to Active Galactic Nuclei (Cambridge: Cambridge Univ. Press)

Salviander, S., Shields, G. A., Gebhardt, K., \& Bonning, E. W. 2007, ApJ, 662 131

Scott, J. E., et al. 2004, ApJ, 615, 135

Shang, Z., Wills, B. J., Wills, D., \& Brotherton, M. S. 2007, AJ, 134, 294

Sigut, T. A. A., \& Pradhan, A. K. 2003, ApJS, 145, 15

Suganuma, M., et al. 2006, ApJ, 639, 46

Sulentic, J. W., Marizani, P., \& Dultzin-Hacyan, D. 2000, ARA\&A, 38, 521

van Zee, L., Salzer, J. J., \& Haynes, M. P. 1998, ApJ, 497, L1

Vanden Berk, D., et al. 2001, AJ, 122, 549

Verner, E., Bruhweiler, F., Verner, D., Johansson, S., \& Gull, T. 2003, ApJ, 592, L59

Wills, B. J., Netzer, H., \& Wills, D. 1985, ApJ, 288, 94

Wills, B. J., Laor, A., Brotherton, M. S., Wills, D., Wilkes, B. J., Ferland, G. J., \& Shang, Z. 1999, ApJ, 515, L53 